\title{
Diversidade de morcegos (Mammalia, Chiroptera) em remanescentes florestais do município de Fênix, noroeste do Paraná, Brasil
}

\author{
Gledson Vigiano Bianconi ${ }^{1,2,4}$, Sandra Bos Mikich ${ }^{3,4}$ \& Wagner André Pedro ${ }^{2}$ \\ 1 Programa de Pós-graduação em Biologia Animal, Universidade Estadual Paulista. Rua Cristóvão Colombo 2265, \\ 15054-000 São José do Rio Preto, São Paulo, Brasil. E-mail: bianconi@terra.com.br \\ ${ }^{2}$ Laboratório de Chiroptera, Departamento de Apoio, Produção e Saúde Animal, Universidade Estadual Paulista. \\ Caixa Postal 341, 16050-680 Araçatuba, São Paulo, Brasil. \\ ${ }^{3}$ Laboratório de Ecologia, Embrapa Florestas. Caixa Postal 319, 83411-000 Colombo, Paraná, Brasil. \\ ${ }^{4}$ Mülleriana: Sociedade Fritz Müller de Ciências Naturais. Caixa Postal 19093, 81531-980 Curitiba, Paraná, Brasil.
}

ABSTRACT. Bat (Mammalia, Chiroptera) diversity in forest remnants of Fênix, State of Paraná, southern Brazil. The richness and the relative abundance of bats were evaluated in three Semideciduous Seasonal Forest fragments located in the municipal district of Fênix, northwest of the State of Paraná, southern Brazil. Between July 2002 and June 2003 bats were sampled with mist-nets set in four 1 ha plots representing different degrees of isolation of riparian and lowland forest. A total of 752 individuals of 14 species of Phyllostomidae $(n=10)$ and Vespertilionidae ( $n=4)$, were captured. Considering the limitations of mist-net sampling, the study area was satisfactorily inventoried based on the ICE estimator. Nevertheless, if compared with similar studies conducted in other Semideciduous Seasonal Forest remnants, the obtained richness is less expressive, suggesting that local extinctions have taken place. Artibeus lituratus (Olfers, 1818) and Carollia perspicillata (Linnaeus, 1758) were dominant in the three studied forest fragments, followed by other three frugivores: A. fimbriatus Gray, 1838, A. jamaicensis Leach, 1821 and Sturnira lilium (E. Geoffroy, 1810). The Shannon's index showed subtle differences among the four studied plots and the Sorensen's index presented high similarities among most of them. However, the grouping analysis revealed higher similarities only between plots representing the same type of vegetation and exhibited two separate groups, one represented by riparian forests and the other by lowland forests, which could be related to particularities in habitat use by bat species. This study also indicated that the forest remnants sampled, in spite of being small, shelter a significant number of bat species and, for that reason, are important for biological conservation.

KEY WORDS. Bat conservation, forest fragmentation, habitat use, Phyllostomidae, Semideciduous Seasonal Forest.

RESUMO. A riqueza de espécies e a abundância relativa de morcegos foram avaliadas em três fragmentos de Floresta Estacional Semidecidual localizados no município de Fênix, noroeste do Estado do Paraná, sul do Brasil. Entre julho de 2002 e junho de 2003 os morcegos foram amostrados com redes-de-neblina instaladas em quatro parcelas de 1 ha cada, representando diferentes graus de isolamento das subformações florestais: aluvial e submontana. Foram capturados 752 exemplares pertencentes a 14 espécies de duas famílias, Phyllostomidae $(\mathrm{n}=10)$ e Vespertilionidae $(\mathrm{n}=4)$. No que se refere a capturas com redes a área foi considerada bem inventariada (estimador ICE). Entretanto, se comparada a estudos similares em Floresta Estacional, a riqueza de espécies foi pouco expressiva, havendo a suspeita que tenham ocorrido perdas de espécies em níveis locais. Artibeus lituratus (Olfers, 1818) e Carollia perspicillata (Linnaeus, 1758) foram numericamente dominantes nos três remanescentes amostrados, seguidas por outros três frugívoros: A. fimbriatus Gray, 1838, A. jamaicensis Leach, 1821 e Sturnira lilium (E. Geoffroy, 1810). O índice de Shannon demonstrou diferenças sutis entre as parcelas amostrais e o índice de Sorensen apresentou alta similaridade entre a maioria delas. Já a análise de agrupamento revelou uma maior afinidade entre parcelas da mesma subformação, exibindo dois grupamentos distintos, um representado pela subformação aluvial e outro pela submontana, sugerindo particularidades no uso do hábitat pelos morcegos. Os resultados indicaram ainda que os remanescentes florestais aqui estudados, apesar de pequenos, abrigam uma parcela significativa das espécies de morcegos esperadas para o bioma e, por essa razão, são importantes para a conservação da diversidade biológica. PALAVRAS CHAVE. Conservação de morcegos, Floresta Estacional Semidecidual, fragmentação florestal, Phyllostomidae, uso de hábitat. 
Os morcegos (Chiroptera, Mammalia) são reconhecidamente importantes na regulação dos ecossistemas tropicais, representando, em algumas áreas, 40 a 50\% das espécies de mamíferos (Patterson \& Pascual 1972, Tımm 1994). Sua notável diversidade de formas, adaptações morfológicas e hábitos alimentares, permite a utilização dos mais variados nichos, em complexa relação de interdependência com o meio (FEnTon et al. 1992, PEDRo et al. 1995, Kalko 1997). À medida que partilham os recursos, em especial os alimentares, os quirópteros influenciam a dinâmica dos ecossistemas naturais, agindo como dispersores de sementes, polinizadores e reguladores de populações animais (RIDLEY 1930, van der PiJl 1957, Goodwin \& Greenhall 1961, Kunz \& Pierson 1994). Ademais, este grupo é um indicador de níveis de alteração no ambiente e bom material de estudo sobre diversidade (FENTON et al. 1992). Desta forma os quirópteros podem ser utilizados como "ferramentas" na identificação dos processos biológicos envolvidos na perda ou transformação do hábitat natural.

Nas últimas três décadas, os avanços nos estudos com morcegos, incluindo aspectos biológicos, biogeográficos, taxonômicos e filogenéticos foram consideráveis (Kunz \& RACEY 1998), contudo, para o Brasil, a base de dados ainda éinsatisfatória (M ARINHo-FILHo \& SAZIMA 1998). O Estado do Paraná reflete bem essa realidade. Em um estudo recente, MIRETZKI (2003) ressaltou que os trabalhos com os morcegos efetuados até o momento no Estado forneceram apenas uma primeira aproximação da real diversidade, havendo extensas áreas com pouca ou nenhuma amostragem. Dentre essas, destaca-se o noroeste paranaense, onde estão local izados os remanescentes que compõem a área de estudo deste trabal ho. Essa região é representada em grande parte pela Floresta Estacional Semidecidual e é uma das mais afetadas pelo processo de fragmentação florestal do Paraná (MAACK 1981, HatSCHBACK \& ZilLeR 1995).

Até o momento, cinco unidades de conservação em florestas estacionais do Paraná foram contempladas com levantamentos de morcegos, a saber: Parque Municipal Arthur Thomas (FéLIX et al. 2001), Parque Estadual Mata dos Godoy (ReIs et al. 1993a, b), Estação Ecológica do Caiuá (MIRETZKI \& MARGARIDO 1999), Parque Estadual do Rio Guarani (Quadros et al. 2000) e Parque Nacional do Iguaçu (SEKı IAmA et al. 2001). Esses estudos conferiram dados sobre biologia, riqueza e abundância relativa dos morcegos, contudo, as informações básicas relacionadas à tolerância e respostas das espécies frente à fragmentação, ainda não foram amplamente discutidas.

Um problema em estudos desta natureza é ausência de informações anteriores à fragmentação que, apesar de não inviabilizarem, dificultam o reconhecimento dosimpactos causados à biota e, em especial, à comunidade de morcegos. Uma maneira de contornar o viés causado por esta deficiência é investir em estudos que detalhem o maior número de áreas e diversifiquem métodos de inventários de fauna. Adicionalmente, o desenho do estudo deve ser pensado a priori, permitindo que os resultados sejam comparáveis entre si e com os disponíveis na literatura. Lamentavelmente, é observada uma extre- ma carência deste tipo de informação em toda Região Neotropical, estando a maioria dos estudos restritos à América do Norte (México, Estrada et al. 1993, Estrada \& Coates-Estrada 2002), América Central (e.g. Guatemala, Schulze et al. 2000) e norte da América do Sul (Guiana Francesa, Brosset et al. 1996, Cosson et al. 1999).

No Brasil extra-amazônico, são poucos os trabalhos a discutir os efeitos da fragmentação dos hábitats na estrutura das comunidades de morcegos. Pedro et al. (1995) analisaram estas questões para um fragmento de $M$ ata Atlântica na cidade de São Paulo. Reis \& Muller (1995) e Reis et al. (2003), trabaIhando em remanescentes florestais de diferentes tamanhos e graus de conservação, avaliaram esses efeitos para o município de Londrina, norte do Estado do Paraná. Nesses estudos os autores sugerem a ocorrência de al terações na composição das comunidades e, em alguns casos, até mesmo extinções locais de espécies.

O estudo aqui apresentado considerou a efetiva necessidade da obtenção de mais informações sobre a estrutura das comunidades de morcegos em áreas fragmentadas. Para tanto, os objetivos bási cos foram os de avaliar a riqueza específica e a abundância relativa da quiropterofauna em três remanescentes da Floresta Estacional do noroeste do Parará, com diferentes graus de isolamento. Essas informações poderão servir como subsídios para o estabelecimento de parâmetros para futuras estratégias de conservação e recuperação da Floresta Estacional Semidecidual da região noroeste paranaense.

\section{Área de estudo}

\section{MATERIAL E MÉTODOS}

Os fragmentos estudados localizam-se no município de Fênix, Estado do Paraná, sul do Brasil (Fig. 1). O clima da região é o subtropical úmido mesotérmico ou Cfa de Köppen, com as médias anuais variando entre $16 \circ \mathrm{C}$ e $29 \circ \mathrm{C}$ (ITCF 1987). A precipitação anual varia entre 1.400 e $1.500 \mathrm{~mm}$, apresentando tendência de concentração de chuvas entre os meses de dezembro e março, sem estação seca definida (MAACK 1981, ITCF 1987). Considerando aspectos fitogeográficos, a região de estudo está inserida nos domínios da Floresta Estacional Semidecidual (sensu VELoso et al. 1992), sendo reconhecidas para região, duas subformações, a Floresta Estacional Semidecidual Submontana (FSM) (áreas mais altas, relativamente distantes dos grandes rios) ea Floresta Estacional Semidecidual Aluvial (FAL) (margens dos rios, sofrendo, em períodos chuvosos, influência de suas cheias). Uma descrição detal hada da vegetação das áreas de estudo pode ser encontrada em MIKICH \& SILvA (2001).

Atualmente, o pouco que resta desta formação florestal na região encontra-se fragmentado, representado por pequenos remanescentes de diferentes tamanhos, estruturas, históricos e usos atuais do solo. Assim, podem ser considerados isolados florestais, tanto pela matriz de áreas cultivadas (principalmente milho, soja e trigo) onde se inserem quanto pela degradação das florestas ciliares (MIKICH \& SILVA 2001). 


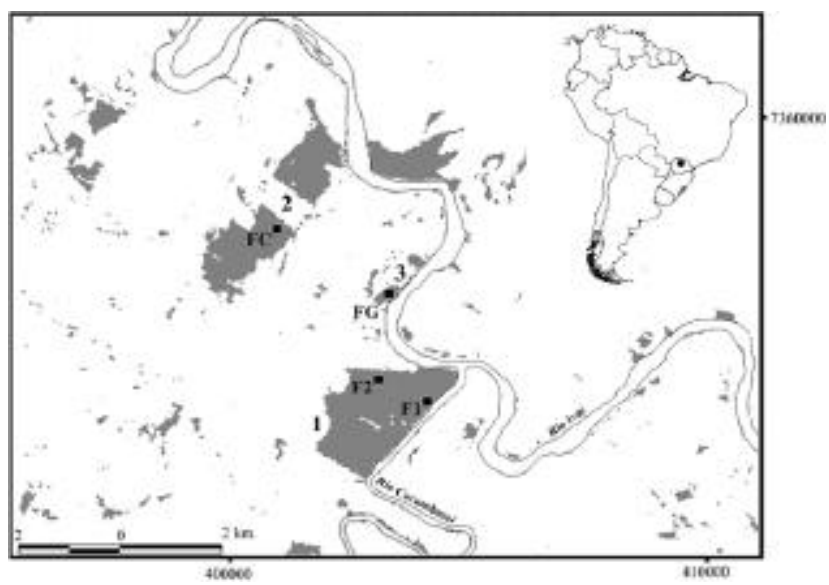

Figura 1. Localização das quatro parcelas amostrais nos três remanescentes de Floresta Estacional Semidecidual do município de Fênix, Estado do Paraná, sul do Brasil, sendo 1 o Parque Estadual Vila Rica do Espírito Santo (parcelas F1 e F2), 2 a Fazenda Cagibi (FC) e 3 a Fazenda Guajuvira (FG).

\section{Parcelas amostrais}

Quatro parcelas amostrais de 1 ha $(100 \times 100 \mathrm{~m})$ foram estabel eci das em três remanescentes florestais: duas no Parque Estadual Vila Rica do Espírito Santo (Parque Vila Rica) (F1 e F2), uma na Fazenda Cagibi (FC) e outra na Fazenda Guajuvira (FG) (Fig. 1).

O Parque Vila Rica (23055'S-5157'W) possui 354 ha de área, formato aproximadamente retangular, estando limitado por áreas de cultivo e pelos rios Ivaí e Corumbataí. Sua maior extensão é ocupada por floresta secundária, mas que em função do tempo de desenvolvimento (aproximadamente 370 anos) assemelha-se em muitos aspectos às florestas primárias alteradas da região (MIKICH \& SILVA 2001). Em função de sua localização, às margens de dois grandes rios, apresenta grandes extensões de FAL, além de FSM. Estas duas subformações apresentam uma faixa de contato de aproximadamente $3 \mathrm{~km}$ de extensão no interior desta unidade de conservação. A FAL possui uma área aproximada de $100 \mathrm{ha}$, estendendo-se por cerca de $300 \mathrm{~m}$ a partir dos rios Ivaí e Corumbataí, em cuja margem foi instalada a parcela F1.

A Fazenda Cagibi (23055'S-5157'W) possui 325 ha defloresta, dividida em duas porções com formatos e dimen sões diferentes. É limitada por áreas cultivadas e pastagens, além do rio Ivaí, que acompanha uma pequena parte da floresta. De uma maneira geral, exibe vários sinais de alteração antrópica, principalmente devido à retirada de madeira de importância econômica (MIKICH \& SILVA 2001). A parcela amostral FC foi instalada na porção representada exclusivamente pela FSM (184 ha).

A Fazenda Guajuvira (235'S-515'' W) possui 24 ha de floresta, estando localizado entre as duas primeiras áreas. É re- presentada em sua totalidade pela FAL, dividida em duas porções de formato e dimensões diferentes, cujos limites são dados por áreas cultivadas, pastagens e pelo rio Ivaí. Provavelmente trata-se de uma floresta secundária, que em alguns locais mistura-se a um pomar (MIKICH \& SILVA 2001). A porção estudada possui uma área de 14 ha e, a exemplo da FAL do ParqueVila Rica, também se estende por aproximadamente 300 $\mathrm{m}$ a partir do rio, em cuja margem foi instalada a parcela FG.

\section{Captura e identificação dos morcegos}

O estudo foi conduzido com periodicidade mensal entre julho de 2002 e junho de 2003 , durante os quais procediam-se capturas durante duas noites em cada uma das quatro parcelas amostrais.

Para a captura dos morcegos foram utilizadas 10 redesde-neblina (12 x 2,5 m), dispostas no centro de cada parcela em trilhas pré-existentes ou abertas para o presente estudo, paralelamente às margens dos rios, intercalando-se redes de malha de 38 e $60 \mathrm{~mm}$. Estas foram abertas ao entardecer, revisadas em interval os de 20 minutos, e fechadas após 6 horas de exposição. Procurando padronizar o esforço de captura em cada parcela amostral as redes não foram fechadas na ocorrência de chuvas e trovoadas. Buscando a al eatoriedade na amostragem, optou-se por sortear a seqüência das parcelas a serem analisadas em cada fase de campo. Os espécimes capturados foram colocados individualmente em sacos de pano e, uma vez identificados, foram anilhados e liberados no mesmo local.

A identificação das espécies seguiu os critérios de Goodwin \& Greenhal (1961), Vizotto \& Taddei (1973), Gonzalez (1989), BARquez et al. (1993), Simmons \& Voss (1998) e Lim \& ENGSTROM (2001). Em complemento, utilizou-se LAVAL (1973) para a identificação das espécies de M yotis Kaup, 1829 e TADDEl et al. (1998) e Ruı et al. (1999) para Artibeus Leach, 1821. O ordenamento taxonômico adotado foi o proposto por Koopman (1993). Uma pequena coleção testemunho foi obtida e depositada no Museu deHistória Natural Capão da Imbuia ( $\mathrm{MHNCI}$ ), Curitiba, Paraná.

\section{A nálise dos dados}

Para obter a relação entre o esforço amostral e o número de espécies registradas foi real izada a multiplicação simples da área de cada rede pelo tempo de exposição, multiplicado também pelo número de repetições e, por fim, pelo número de redes, conforme proposta apresentada por Straube \& Bianconı (2002).

Curvas de rarefação foram utilizadas para comparar o acúmulo de espécies, relacionando-o com o número de exemplares obtidos e o esforço amostral. Para extrapolar os dadose, assim, avaliar quão completo foi o inventário no que se refere ao método de coleta empregado, foi utilizado um estimador de espécies baseado na cobertura (“ICE”, COLWELL 2001).

Foram utilizados, para a análise de diversidade e uniformidade, os índices de diversidade de Shannon-Wiener $\left(\mathrm{H}^{\prime}\right)$ e Pielou (e) (Odum 1988), respectivamente, considerando somente a primeira captura de cada indivíduo. Para comparar a simila- 
ridade das parcelas foi utilizado o índice de similaridade de Sorensen (LudwIG \& REYNoLds 1988). Utilizou-se para comparações estatísticas entreas áreas o teste de proporção (TRIOLA 1999). A constância (C) foi calculada (Silveira-Neto et al. 1976), sendo as espécies classificadas como comuns na amostragem $(C \geq$ $50 \%$ ), relativamente comuns ( $25 \leq \mathrm{C}<50 \%$ ) e raras ( $<25 \%)$.

\section{RESULTADOS}

\section{Capturas}

Foram obtidas 752 capturas de morcegos (incluindo 54 recapturas), representando 14 espécies de duas famílias: Phyllostomidae e Vespertilionidae. O maior número de capturas ocorreu na Fazenda Cagibi (208 indivíduos), seguido pelas parcelas F2 (197) e F1 (192) do Parque Vila Rica e por último pela Fazenda Guajuvira (155) (Tab. I). Essas diferenças foram significativas entre as fazendas Guajuvira e Cagibi $(Z=-3,19 ; p$ $\leq 0,001$ ) e entre a Fazenda Guajuvira e ambas as parcelas (F1 e F2) do Parque Vila Rica ( $Z=-2,26 ; p=0,01 ; Z=-2,56 ; p=0,005$, respectivamente).

Foram registradas 12 espécies de morcegos para o Parque Vila Rica, nove na parcela F2 e 10 na F1, sendo, nesta última, duas exclusivas: M yotis aff. riparius Handely, 1960 eVampyressa pusilla (Wagner, 1843). A Fazenda Guajuvira apresentou 10 espécies, uma exclusiva (Chiroderma villosum Peters, 1860) e a Fazenda Cagibi nove, com uma exclusiva (M icronycteris megalotis (Gray, 1842)) (Tab. I).

O maior número de espécies por capturas foi observado para a Fazenda Guajuvira (0,064 espécies/capturas), seguido pelo Parque Vila Rica na F2 $(0,050)$ e na F1 $(0,046)$ e pela Fazenda Cagibi $(0,043)$ (Tab. I).

A relação entre o esforço amostral total nas quatro parcelas ( $172.800 \mathrm{~m}^{2}$.h) e o número de táxons registrados foi de 0,81 espécies para cada $10.000 \mathrm{~m}^{2}$.h. Verificou-se um rápido acúmulo de espécies no início do estudo, com oito novos registros quando transcorridos apenas 18,7\% (32.400 $\left.\mathrm{m}^{2} . \mathrm{h}\right)$ do esforço total de captura. Duas novas espécies foram acrescentadas quase ao final do trabalho (Chiroderma villosum e Vampyressa pusilla), quando mais de $85 \%$ do esforço de captura já havia sido cumprido (Fig. 2). Esse resultado, embora de forma menos conspícua, repete-se na análise de cada parcela amostral individualmente.

A curva do coletor baseada no número de exemplares obtidos, não indicou uma assíntota definida ao final das capturas (Fig. 3). Alguns procedimentos estatísticos podem ser utilizados para extrapolação dos dados e, assim, avaliar quão completo foi o inventário no que se refere ao método empregado. Utilizando um estimador de espécies baseado na cobertura ("ICE", Colwell 2001), que representa a soma das probabilidades de encontro de cada espécie observada, verificou-se que a partir da amostra nove, a curva começa a mostrar um comportamento assintótico, ou seja, por volta de 17 espécies (Fig. 4).

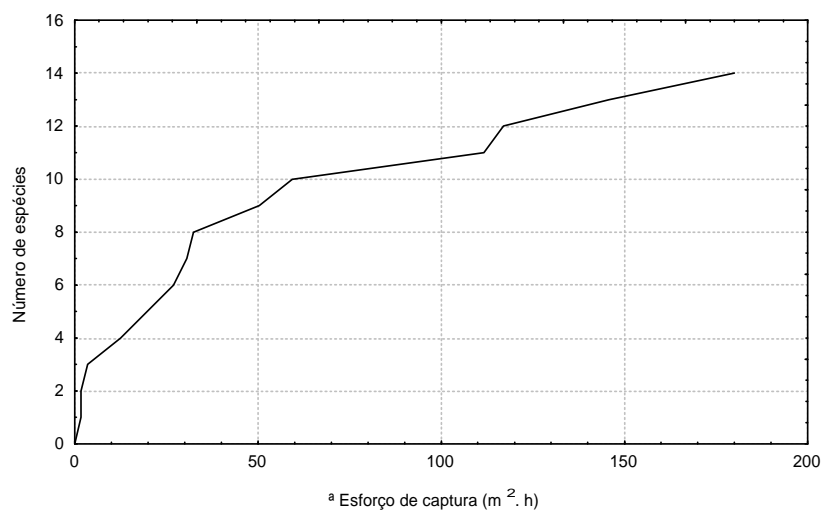

Figura 2. Número cumulativo de espécies registradas em função do esforço de captura em três remanescentes florestais do município de Fênix, Estado do Paraná, Brasil amostrados entre julho de 2002 e junho de 2003. a Valores na base $10^{3}$.

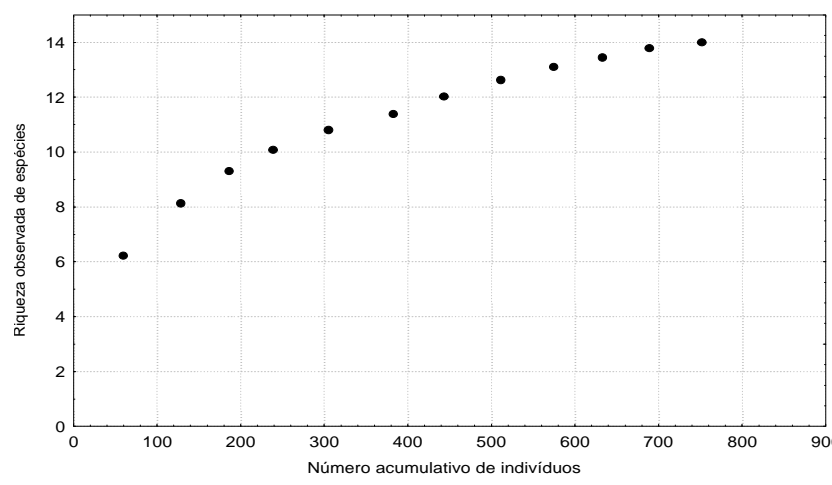

Figura 3. Riqueza observada de espécies (curva do coletor) em função do número acumulativo de exemplares em três remanescentes florestais do município de Fênix, Estado do Paraná, Brasil, amostrados entre julho de 2002 e junho de 2003.

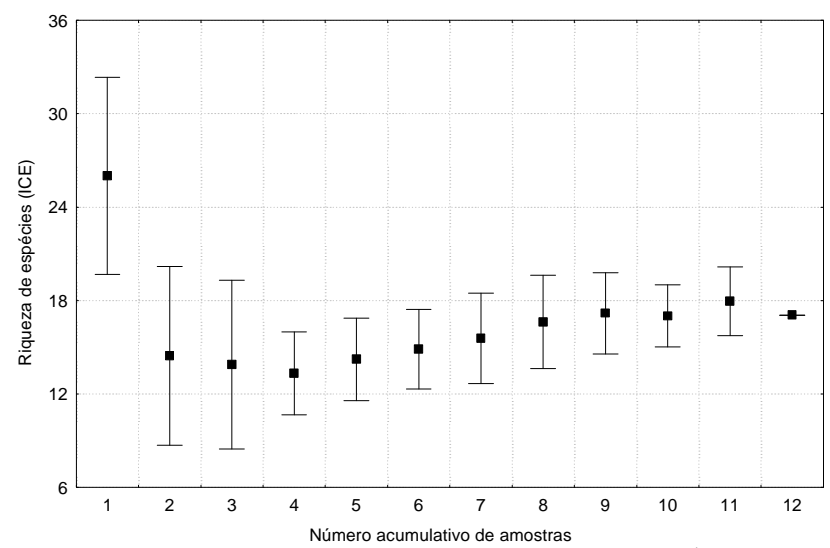

Figura 4. Número de espécies estimadas com o ICE (e erro padrão) em função do número acumulativo de amostras, em três remanescentes florestais do município de Fênix, Estado do Paraná, Brasil, amostrados entre julho de 2002 e junho de 2003. 
Tabela I. Relação das capturas efetuadas entre julho de 2002 e junho de 2003, em quatro parcelas amostrais de um hectare, localizadas no município de Fênix, Paraná. (a) Número de espécies dividido pelo número de capturas.

\begin{tabular}{ccccccc}
\hline Parcelas & $\begin{array}{c}\text { Esforço } \\
\text { de captura (E) }\end{array}$ & $\begin{array}{c}\text { Número } \\
\text { de capturas }\end{array}$ & $\begin{array}{c}\text { Porcentagem } \\
\text { de capturas }\end{array}$ & $\begin{array}{c}\text { Número de } \\
\text { espécies }\end{array}$ & $\begin{array}{c}\text { Espécies } \\
\text { exclusivas }\end{array}$ & $\begin{array}{c}\text { Proporção } \\
\text { de espécies }\end{array}$ \\
\hline F1 & $43,2.10^{3} \mathrm{~m}^{2} . \mathrm{h}$ & 192 & 25,5 & 9 & 2 & 0,046 \\
F2 & $43,2.10^{3} \mathrm{~m}^{2} . \mathrm{h}$ & 197 & 26,2 & 10 & 0 & 0,050 \\
FC & $43,2.10^{3} \mathrm{~m}^{2} . \mathrm{h}$ & 208 & 27,7 & 9 & 10 & 0,043 \\
FG & $43,2.10^{3} \mathrm{~m}^{2} . \mathrm{h}$ & 155 & 20,6 & 14 & 4 & 0,064 \\
\hline Total & $172,8.10^{3} \mathrm{~m}^{2} . \mathrm{h}$ & 752 & 100 & 0,018 \\
\hline
\end{tabular}

\section{Composição das espécies e padrões de abundância}

Osfilostomídeos, com 10 espécies de quatro subfamílias, representaram $97,6 \%$ das capturas. Os quatro exemplares restantes são vespertilionídeos (Tab. II). Individualmente, os filostomídeos representaram $98,7 \%$ do total de capturas de FG, $98,1 \%$ de FC, $97,4 \%$ para F1 e $96,4 \%$ para F2.

Artibeus lituratus (OIfers, 1818) foi a espécie mais capturada, representando $54,9 \%$ da amostragem total, seguido por Carollia perspicillata (Linnaeus, 1758) (19,7\%), A. fimbriatus Gray, 1842 (8,6\%), Sturnira lilium (E. Geoffroy, 1810) (6,6\%) e A. jamaicensis Leach, 1821 (6,1\%). Juntas, estas espécies representaram $95,9 \%$ do total de morcegos obtidos, cabendo ao restante (oito espécies), somente $4,1 \%$ do total de capturas (Tab. II). Em todas as parcelas ocorreu o predomínio de A. lituratus sobre $C$. perspicillata, sendo que a menor diferença nessa proporção foi verificada para a FAL (F1 e FG).

Calculado o índice de constância (SILVEIRA-Neto et al. 1976) foram encontradas seis espécies comuns: Artibeus lituratus, A. fimbriatus, A. jamaicensis, Sturnira lilium, Carollia perspicillata e Myotis nigricans (Schinz, 1821), e uma relativamente comum: Desmodus rotundus (E. Geoffroy, 1810). Todas as demais espécies foram consi deradas raras na amostragem: Chrotopterus auritus (Peters, 1856), Chiroderma villosum, Micronycteris megalotis, Vampyressa pusilla, Lasiurus blossevillii (Müller, 1776), Myotis aff. riparius e M yotis levis (I. Geoffroy, 1824).

Diversidade de espécies e similaridade entre as parcelas

A diversidade de espécies calculada pelo índice de Shannon-Wiener $\left(\mathrm{H}^{\prime}\right)$ para os três remanescentes foi de 1,38 e a uniformidade (e) de 0,52. Individualmente, as parcelas F1 e F2 do Parque Vila Rica apresentaram maior diversidade e uniformidade, seguidas pelas parcelas FC e FG (Tab. III).

A similaridade entre as parcelas, cal culada pelo índice de Sorensen, al cançou valores el evados entre F1 e FG e entre F2 e FC. As duas parcelas alocadas no interior do Parque Vila Rica também apresentaram alta similaridade. A menor similaridade ocorreu entre as parcelas FC e FG (Tab. IV).

Em concordância, a análise de Cluster baseada na freqüência de ocorrência das espécies, produziu dois grupos distintos, estando o primeiro representado por F1 e FG e o segundo por F2 e FC (Fig. 5).
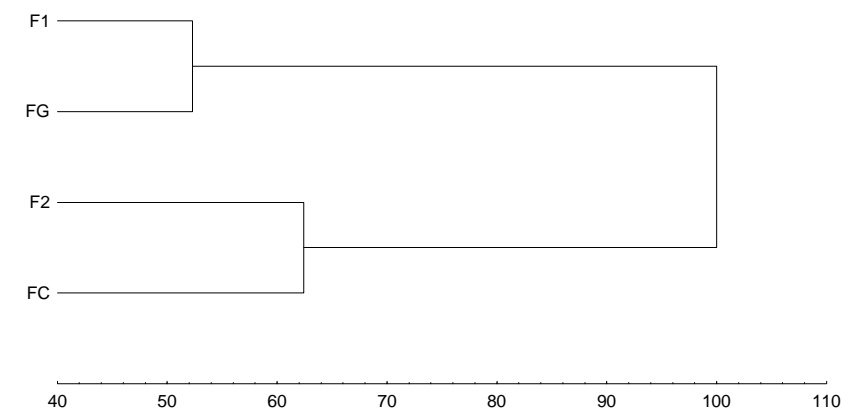

Figura 5. Dendograma originado da análise de agrupamento da matriz de similaridade para quatro parcelas amostrais de um hectare, localizadas no município de Fênix, Estado do Paraná, Brasil. Método UPGMA com distâncias Euclidianas.

\section{DISCUSSÃ O}

\section{Riqueza de espécies e padrões de abundância}

Considerando-se os táxons registrados neste estudo e aquel es col etados anteriormente na mesma local idade (Phyllostomus hastatus e Eptesicus brasiliensis) (G.V. Bianconi, obs. pess.), somaram-se 16 espécies. De acordo com MIRETZKI (2003), um inventário com esse número de espécies de morcegos, definiria a localidade como "razoavelmente conhecida". O valor obtido representa $26,4 \%$ das espécies registradas para o Paraná e 35,9\% das esperadas para a Floresta Estacional paranaense (MIRETZKI 2003). Ainda segundo esse autor, este tipo fitofisionômico é, até o momento, o mais rico em espécies no Estado, com 39 espécies de quatro famílias: Noctilionidae (1 espécie), Phyllostomidae (20), Vespertilionidae (8) e Molossidae (10).

Os filostomídeos predominaram na área de estudo, com 11 espécies. Os morcegos desta família são endêmicos da região Neotropical eo grupo geral mente predominantee de maior diversidade nas comunidades de mamíferos (HUMPHREY \& Bonaccorso 1979, Fenton et al. 1992, Koopman 1993). Isso foi demonstrado nos muitos trabal hos realizados em áreas de florestas neotropicais, on de cerca de $40 \%$ das espécies dessa família podem ocorrer em sintopia ou simpatria (e.g. Brosset \& Charles-Dominique 1990, Simmons \& Voss 1998). 
Tabela II. Espécies, número e abundância relativa de morcegos capturados entre julho de 2002 e junho de 2003 em quatro parcelas amostrais de um hectare localizadas no município de Fênix, Paraná, Brasil.

\begin{tabular}{|c|c|c|c|c|c|}
\hline \multirow{2}{*}{ Ordenamento Taxonômico } & \multicolumn{4}{|c|}{ Número de indivíduos capturados/parcela } & \multirow{2}{*}{ Total } \\
\hline & $\mathrm{F} 1$ & $\mathrm{~F} 2$ & $\mathrm{FC}$ & FG & \\
\hline \multicolumn{6}{|l|}{ Phyllostomidae } \\
\hline \multicolumn{6}{|l|}{ Phyllostominae } \\
\hline Chrotopterus auritus (Peters, 1856) & 0 & $1(0,5)$ & $1(0,5)$ & $1(0,6)$ & $3(0,4)$ \\
\hline M icronycteris megalotis (Gray, 1842) & 0 & 0 & $1(0,5)$ & 0 & $1(0,1)$ \\
\hline \multicolumn{6}{|l|}{ Carollinae } \\
\hline Carollia perspicillata (Linnaeus, 1758) & $43(22,4)$ & $35(17,8)$ & $21(10,1)$ & $49(31,6)$ & $148(19,7)$ \\
\hline \multicolumn{6}{|l|}{ Stenodermatinae } \\
\hline Artibeus fimbriatus Gray, 1838 & $19(9,9)$ & $19(9,6)$ & $18(8,7)$ & $9(5,8)$ & $65(8,6)$ \\
\hline Artibeus jamaicensis Leach, 1821 & $12(6,3)$ & $14(7,1)$ & $15(7,2)$ & $5(3,2)$ & $46(6,1)$ \\
\hline Artibeus lituratus (Olfers, 1818) & $91(47,4)$ & $112(56,9)$ & $129(62,0)$ & $81(52,3)$ & $413(54,9)$ \\
\hline Chiroderma villosum Peters, 1860 & 0 & 0 & 0 & $1(0,6)$ & $1(0,1)$ \\
\hline Sturnira lilium (E. Geoffroy, 1810) & $19(9,9)$ & $6(3,0)$ & $19(9,1)$ & $6(3,9)$ & $50(6,6)$ \\
\hline Vampyressa pusilla (Wagner, 1843) & $2(1,0)$ & 0 & 0 & 0 & $2(0,3)$ \\
\hline \multicolumn{6}{|l|}{ Desmodontinae } \\
\hline Desmodus rotundus (E. Geoffroy, 1810) & $1(0,5)$ & $3(1,5)$ & 0 & $1(0,6)$ & $5(0,7)$ \\
\hline \multicolumn{6}{|l|}{ Vespertilionidae } \\
\hline \multicolumn{6}{|l|}{ Vespertilioninae } \\
\hline Lasiurus blossevillii (Müller, 1776) & 0 & $1(0,5)$ & $1(0,5)$ & 0 & $2(0,3)$ \\
\hline Myotis levis (I. Geoffroy, 1824) & 0 & $1(0,5)$ & 0 & $1(0,6)$ & $2(0,3)$ \\
\hline Myotis nigricans (Schinz, 1821) & $4(2,1)$ & $5(2,5)$ & $3(1,4)$ & $1(0,6)$ & $13(1,7)$ \\
\hline Myotis aff. riparius Handley, 1960 & $1(0,5)$ & 0 & 0 & 0 & $1(0,1)$ \\
\hline Total & $192(100)$ & $197(100)$ & $208(100)$ & $155(100)$ & $752(100)$ \\
\hline
\end{tabular}

Tabela III. Índices de diversidade de Shannon-Wiener ( $\left.\mathrm{H}^{\prime}\right)$ e de uniformidade de Pielou (e) para três remanescentes florestais localizados no município de Fênix, Estado do Paraná, Brasil.

\begin{tabular}{lcc}
\hline \multicolumn{1}{c}{ Local } & Diversidade (H') & Uniformidade (e) \\
\hline Fazenda Cagibi (FC) & 1,27 & 0,58 \\
Fazenda Guajuvira (FG) & 1,23 & 0,56 \\
Parque Vila Rica (F1 e F2) & 1,49 & 0,60 \\
FAL Parque Vila Rica (F1) & 1,47 & 0,70 \\
FSM Parque Vila Rica (F2) & 1,37 & 0,59 \\
\hline
\end{tabular}

Levantamentos de morcegos realizados na Floresta Estacional paranaensetambém demonstram predominância de filostomídeos sobre as espécies das demais famílias (Reıs et al. 1993a, MiretZki \& Margarido 1999, Félix et al. 2001, SeKIAMA et al. 2001). A ausência e/ ou baixa diversidade das outras famílias possivelmente se deve à seletividade das redes-de-neblina método que favorece a captura de morcegos que se deslocam
Tabela IV. Índice de similaridade de Sorensen (S) obtido entre quatro parcelas amostrais de um hectare, localizadas no município de Fênix, Estado do Paraná, Brasil.

\begin{tabular}{ccccc}
\hline & F1 & F2 & FC & FG \\
\hline F1 & 1,00 & & & \\
F2 & 0,86 & 1,00 & & \\
FC & 0,82 & 0,86 & 1,00 & \\
FG & 0,84 & 0,81 & 0,70 & 1,00 \\
\hline
\end{tabular}

principalmente pel o sub-bosque, permitindo uma amostragem abundante de filostomídeos (GreEnHALL \& Paradiso 1968, LAVAL \& Fitch 1977, Trajano 1984, Pedro \& Taddel 1997, Straube \& BIANCONI 2002).

Desse modo, podemos considerar a área de estudo como bem inventariada somente no que se refere a capturas com redes (estimador ICE $=17$ espécies), pois a utilização de outras 
técnicas de amostragem (ver Kunz \& KURTA 1990) deverá registrar novas espécies de morcegos para a localidade, especial mente vespertilionídeos e molossídeos. Não obstante, o uso de redes neste trabalho, assim como em todos os inventários disponíveis para Floresta Estacional, permitiu a reunião das informações para compor as análises comparativas subseqüentes.

Se analisarmos alguns trabalhos similares em Floresta Estacional, o número de espécies observadas ( $n=14$ ) parece, a princípio, pouco expressivo (e.g. Reıs et al. 1993b - 27 espécies, Sekiama et al. 2001 - 26, Pedro et al. 2001 - 23). Contudo, para verificar o real significado desta riqueza e o que ela representa da comunidade de morcegos da área, algumas considerações devem ser feitas.

Determinadas áreas amostradas de mesma formação florestal, mas com maiores extensões, têm demonstrado uma evidente superioridade na riqueza de quirópteros. O mel hor exemplo é o do Parque Nacional do Iguaçu, no sudoeste do Paraná, com uma área superior a 170 mil hectares (IBDF 1981). Neste Parque, Sekiama et al. (2001) registraram 26 espécies em 1403 capturas. Números parecidos também foram obtidos em áreas menores, porém significativamente mais extensas que os remanescentes estudados. Entre elas temos a Estação Ecológica de Caetetus (2.178 hectares), centro-oeste de São Paulo, com 23 espécies em 468 capturas (PEDRo et al. 2001) e o Parque Estadual Mata dos Godoy (680 hectares), norte do Paraná, com 27 espécies em 971 capturas (Reis et al. 1993b). Ainda nesse sentido, chamam a atenção os resultados de MIRETZKI \& MARGARIDO (1999) para a Estação Ecológica do Caiuá (1.427 hectares), noroeste do Paraná. Neste estudo, os autores registraram 14 espécies em apenas 66 capturas, sendo estas representantes das quatro famílias de morcegos esperadas para o bioma.

Os fragmentos do município de Fênix representam uma porção ínfima da Floresta Estacional paranaense, a qual ocupava $46 \%$ do território do Estado (MAACK 1981). Devido à devastação do bioma nessa localidade, hoje representada apenas por pequenos remanescentes (MIKICH \& SILVA 2001), suspeita-se que tenham ocorrido perdas de espécies em níveis locais. Alguns estudos nos trópicos têm demonstrado reduções na abundância e na riqueza de morcegos quando da al teração antrópica dos hábitats (e.g. Reis \& Muller 1995, Pedro et al. 1995, WiLson et al. 1996, Cosson et al. 1999, Rels et al. 2002). Para a Floresta Estacional do norte do Paraná, por exemplo, ReIs \& MuLLeR (1995) e Ress et al. (2002) sugerem que o pequeno tamanho de alguns fragmentos florestais do município de Londrina, acarretou alterações na composição dos conjuntos taxonômicos, podendo estas áreas, até mesmo, não sustentarem populações de algumas espécies de quirópteros.

As comunidades de morcegos neotropicais apresentam algumas espécies dominantes co-existindo com várias raras (Trajano 1984, Pedro \& Taddel 1997). Neste estudo, duas espécies de filostomídeos, Artibeus lituratus (Stenodermatinae) e Carollia perspicillata (Carollinae), foram numericamente dominantes nos três fragmentos florestais amostrados. Considera- dos comuns no neotrópico (FLeming 1988, Brosset \& CharlesDominique 1990, Pedro et al. 2001), esses frugívoros representam grande parte das capturas em estudos na Floresta Estacional paranaense (M uller \& ReIs 1992, Reis et al. 1993a, 1999, SeKIAMA et al. 2001, MIRETZKI 2003).

Alguns autores sugerem que A. lituratus e C. perspicillata possuem uma maior eficiência em adaptarem-se aos processos de fragmentação e/ou modificação do hábitat (BRosset et al. 1996, Wilson et al. 1996, Estrada \& Coates-Estrada 2002). A presença dessas espécies tem se mostrado especial mente abundante em áreas alteradas (MuLler \& ReIs 1992, WILSON et al. 1996, MiretzKI \& Margarido 1999, Pedro et al. 2001), incluindo até pequenas manchas florestais no interior de grandes áreas urbanas (Félix et al. 2001, ReIs et al. 2003). Segundo Estrada \& CoATEs-Estrada (2002), tal flexibilidade pode estar relacionada com sua capacidade de utilizar vários estratos da vegetação, beneficiando-se das diversas oportunidades presentes nos ambientes modificados pelo homem.

Outras três espécies abundantes nos fragmentos estudados foram os Stenodermatinae: A. fimbriatus, A. jamaicensis e Sturnira lilium. Em al gumas regiões do sul do Brasil A. fimbriatus é considerada a segunda espécie mais abundante de Phyllostomidae, depois de A. lituratus (FÁBIAn et al. 1999). No Paraná, destaca-se como um dos frugívoros mais capturados em al guns estudos na Floresta Estacional, como a Estação Ecológica de Caiuá (MIRETZKI \& M ARGARIDo 1999), o Parque Estadual Mata dos Godoy (Reis et al. 2000) e o Parque Nacional do Iguaçu (SekıAmA et al. 2001). Seu congênere A. jamaicensis, é capturado com menor freqüência (Reıs et al. 2002), não aparecendo, por vezes, em estudos no bioma (e.g. Miretzkı \& Margarido 1999, Sekiama et al. 2001). Sturnira lilium é um dos táxons mais capturados em fragmentos florestais das regiões sul e sudeste do Brasil (SIPInSKI \& ReIs 1995, RuI \& FábIAn 1997, Fábian et al. 1999, Reis et al. 1996, Taddei \& Pedro 1998, Bianconi et al. 2003), incluindo áreas de Floresta Estacional (M ULLeR \& ReIs 1992, Pedro et al. 2001, FéLIx et al. 2001).

Ainda que os fragmentos estudados possuam pequenas dimensões, estes ainda mantêm uma elevada abundância de recursos alimentares (cf. MIKICH \& SILVA 2001) freqüentemente citados para dieta das subfamílias Stenodermatinae Carollinae, como os representantes dos gêneros Ficus L. (Moraceae), Cecropia Loefl. (Cecropiaceae), Piper L. (Piperaceae) e Solanum L. (Solanaceae) (Reis \& Perachi 1987, Fleming 1988, Palmeirim et al. 1989, Handley et al.1991, ZortÉA \& ChiARELLo 1994, SiPInSKI \& Reis 1995, Reis et al. 1996, Wendeln et al. 2000, MIKICH 2002, Nogueira \& Peracchi 2002, Passos et al. 2003).

Outra consideração refere-se à disposição dos fragmentos florestais na matriz cultivada. A pequena distância entre os remanescentes (Fig. 1) potencializa sua utilização de forma conjunta por al gumas espécies de frugívoros, conforme observado por Law \& Dickman (1998) e Estrada \& Coates-Estrada (2002). Morcegos do gênero Artibeus, por exemplo, que se alimentam preferencialmente de Ficus spp., costumam voar lon- 
gas distâncias em busca de alimento (e.g. Heithaus et al. 1975, BonNACORSO 1979, HANDLEY \& MoRRISON 1991). Dada a redução da disponibilidade deste recurso em paisagens fragmentadas, esse comportamento pode, em hipótese, ser ainda mais acentuado (StoCKWELL 2001).

As espécies Vampyressa pusilla eChiroderma villosum foram consideradas raras na amostragem. No Paraná, V. pusilla foi registrada para as regiões norte e sudeste do Estado, sendo normalmente representada por poucos indivíduos (REIS \& MULLER 1995, MIRETZKI 2003). O registro de $C$. villosum representa a segunda ocorrência para o Estado. Tanto C. villosum quanto V. pusilla têm os frutos de Ficus spp. como item principal em sua dieta (Bonaccorso 1979, Reis et al. 1996, Pedro et al. 1997, Wendeln et al. 2000, Nogueira \& Peracchi 2002). Essa característica, no entanto, é ainda mais intensa no que se refere a $C$. villosum, estando sua ocorrência em determinadas áreas associada à disponibilidade de Ficus (Nogueira \& Perach 2002). Para os remanescentes estudados, Ficus spp. é considerado um recurso relativamente comum (MIKICH \& SILVA 2001), ressaltando, desta forma, a importância dos fragmentos para a conservação da espécie.

A captura de Chrotopterus auritus - morcego de grande porte que inclui pequenos vertebrados em sua dieta (GARDNER 1977) - foi rara na área. Em estudos similares, a espécie também foi capturada em baixa freqüência (e.g. Reıs et al. 1993a, Taddel \& Pedro 1998, Félix et al. 2001 Pedro et al. 2001). Ainda entre os Phyllostominae ocorreu o registro de Micronycteris megalotis, também rara em muitos estudos (e.g. Rels et al. 2000, Félıx et al. 2001). Muitas espécies desta subfamília parecem ser sensíveis à fragmentação, podendo a sua presença em determinada área ser um bom indicativo da integridade do ambiente (FEnTon et al. 1992, WILson et al. 1996).

Desmodus rotundus, considerado relativamente comum na Floresta Estacional (e.g. Reis \& MulLer 1995, Reis et al. 2000, PEDRo et al. 2001), é capturado com freqüência em áreas abertas, florestas fragmentadas ou contínuas (BonacCorso 1979, Fenton et al. 1992, Simmons \& Voss 1998). Estrada \& CoatesEstrada (2002) sugerem que a espécie utiliza áreas florestais como abrigo e/ou stepping stones (trampolins ecológicos) quando da busca por alimento em áreas de pastagens. Embora a matriz alterada onde os fragmentos estão inseridos seja representada em sua maior parte por plantações, alguns eqüinos e bovinos podem ser encontrados nas propriedades, servindo assim de recurso alimentar ao vampiro.

Vespertilionidae, com exceção de Myotis nigricans, foi rara na amostragem (Lasiurus blossevillii, Myotis levis e Myotis aff. riparius). Ainda que estetenha sido o padrão observado em vários estudos na Floresta Estacional (e.g. Reıs et al. 1993a, 2000, FéıIx et al. 2001), qualquer análise mais criteriosa envolvendo este táxon fica comprometida devido à sel etividade das redes-de-neblina.

\section{Os fragmentos florestais e suas subformações}

Florestas bem estruturadasten dem a concentrar uma quantidade superior de recursos potencialmente úteis e valiosos aos morcegos (Vonhof \& Barclay 1996 ERICKSON \& W EST 2003). O Par- que Vila Rica (354 ha) ea Fazenda Cagibi (325 ha) estão entre os maiores e mais bem preservados remanescentes florestais do município deFênix. Dadas sua extensão e características vegetacionais relacionadas a florestas mais ou menos desenvolvidas (MIKICH \& SILVA 2001), essas áreas parecem fornecer um maior suporte para as comunidades de morcegos, determinando maior complexidade na composição da comunidade. De fato, as parcelas alocadas no interior desses fragmentos, além de apresentarem número de capturas significativamente superiores àqueles obtidos na Fazenda Guajuvira (24 ha), indicaram um maior valor no índice de Shannon-Wiener e uma maior uniformidade.

Segundo Pedro \& TAdDel (1997), há constância na diversidade de conjuntos taxonômicos de morcegos, medidas pelo índice de Shannon, ao redor de 2,0 (e.g. Pedro et al. $2001-\mathrm{H}^{\prime}$ 2,26, Pedro \& Taddei 1997 - 2,11, Estrada \& Coates-Estrada 2002 - entre 2,3 e 2,5). Geral mente, a riqueza em espécies de morcegos é significativamente maior em áreas conservadas do que em áreas alteradas (FENTON et al. 1992). Partindo deste princípio e, em comparação dos valores $H^{\prime}$ deste estudo com os valores obtidos para outros conjuntos taxonômicos, podemos sugerir que o processo de fragmentação florestal no município deFênix pode ter gerado uma simplificação na comunidade de quirópteros dos remanescentes estudados.

No que se refere ao número de espécies registradas para as áreas, o Parque Vila Rica, com 12 táxons, foi a que melhor representou a quiropterofauna da região. Para justificar essa condição, duas abordagens distintas devem ser feitas. A primeira relaciona-se ao fato de haver duas parcelas amostrais no Parque, resultando num esforço de captura maior do que aquele aplicado às fazendas Cagibi e Guajuvira e, por conseguinte, uma amostragem mais completa. Outro aspecto a ser considerado é o Parque ser o maior dentre os três remanescentes estudados, ser bem conservado, e a representar ainda, de forma contínua, duas subformações florestais (FAL e FSM).

Por outro lado, quando da análise do número de espécies por parcela amostral, não foram detectadas grandes variações, inclusive no que se refere a espécies exclusivas, que, por serem raras na amostragem, não forneceram indícios suficientes que comprovassem preferência a um determinado hábitat. O fato dea Fazenda Guajuvira ter apresentado uma maior média de espécies/captura, pode estar relacionado ao pequeno tamanho do remanescente, permitindo, quando da instalação das redes-de-neblina, amostrar uma porção expressiva da área total do fragmento.

Quanto aos padrões de captura das espécies nas parcelas, destacam-se os dados obtidos para Carollia perspicillata. Conforme observado, as áreas de FAL (F1 e FG) representaram o maior número de capturas e também a maior abundância relativa deste frugívoro, quando comparadas às áreas de FSM (F2 e FC). Segundo Fleming \& Heithaus (1986), os principais fatores a influenciar o comportamento de forrageio de $C$. perspicillata são, em grau de importância, a distribuição do alimento, o ris- 
co de predação ea hierarquia social. A base de dados hoje disponíveis para a espécie, bem como para as subformações florestais, dificulta a avaliação destas variáveis. Por exemplo: ainda que sai bamos que os fragmentos florestais apresentam nove espécies de Piper, frutos preferencialmente consumidos por $C$. perspicillata (FLEMING 1985, PALMEIRIM et al. 1989, MIKICH 2002) e distribuídos abundantemente ao longo de estradas e trilhas, borda e interior da floresta (MIKICH \& SILVA 2001), não podemos afirmar que as parcelas de FAL concentram uma quantidade superior deste recurso, comparada à FSM. Assim, uma mel hor compreensão desses fatores só será possível com a continuidade dos trabalhos, que devem prever a realização de um levantamento quali-quantitativo da disponibilidadedefrutosquiropterocóricos em cada subformação florestal, bem como o refinamento das informações bionômicas de C. perspicillata para área.

Ao contrário do esperado, o índice de Sorensen indicou alta similaridade entre a maioria das parcelas amostrais, inclusive entre parcel as local izadas em diferentes subformações florestais, como F1 e F2. Neste caso, no entanto, devido ao fato da FAL e FSM, representadas por estas parcel as, apresentaremse contíguas dentro do Parque Vila Rica, o deslocamento de morcegos entre elas deve ter sido facilitado, contribuindo para a sua similaridade. Esta hipótese pode ser corroborada pela diferença observada no índice obtido para FC x FG. Neste último caso, as subformações não são contíguas e foram encontradas diferenças relativamente altas na freqüência de captura de algumas espécies, especialmente Carollia perspicillata, conforme discutido acima. De fato, a análise de agrupamento também sugere uma maior afinidade entre parcelas da mesma subformação, exibindo dois grupamentos distintos, representados pelas subformações, que devem ser provocados por particularidades no uso do hábitat pelos morcegos.

Vale ressaltar que, apesar da diversidade ser supostamente inferior a de outras taxocenoses estudadas, havendo inclusive a suspeita de perdas de espécies em níveis locais, os remanescentes de Floresta Estacional Semidecidual do município de Fênix ainda abrigam uma parcela significativa das espécies de morcegos esperadas para o bioma, incluindo até mesmo táxons considerados raros.

A indicação de que pequenos fragmentos florestais, como a Fazenda Guajuvira, são fundamentais à manutenção de muitas espécies da comunidade, ressalta ainda mais a importância da recuperação e restabelecimento da FAL ao longo dos grandes rios da região para conservação da quiropterofauna.

\section{AGRADECIMENTOS}

Ao FEM A/IAP/SEMA (conv. 024/02) e ao Mater Natura Instituto de Estudos Ambientais pelo auxílio financeiro; ao CNPq pela bolsa para Wagner André Pedro (300845/98-7) e à CAPES pela bolsa para Gledson Vigiano Bianconi. Somos gratos à FAPESP (processos 98/12556-1 e 98/08940-0) eà Embrapa Florestas pelo apoio; aos funcionários do IAP e do Parque Vila Rica pela ajuda e uso das dependências; aos proprietários e administradores das fazendas Cagibi e Guajuvira pela autorização de trabalho; a Fabiana Rocha Mendes, Daniel Carvalho Carneiro, Carlos Eduardo Conte, Arthur A. Bispo de Oliveira, Rodrigo P. Napoli e Gustavo Graciolli pelo auxílio nas atividades de campo. A Michel Miretzki, Marlon Zortéa, Lílian Casatti, Vinícius Abilhoa, Fabiana Rocha Mendes e Fernando Costa Straube pelas críticas e contribuições à versão preliminar desse texto, bem como a Fernando de Camargo Passos, Nélio Roberto dos Reis e um revisor anônimo pelas sugestões. A Alberto Urben Filho e Michel Miretzki pela confecção do mapa; e a Ana Eugênia C. Campos-Farinha pela revisão do abstract.

\section{REFERÊNCIAS BIBLIOGRÁ FICAS}

Barquez, R.M.; N.P. GIanninI \& M.A. M ARes. 1993. Guide to the Bats of Argentina/ Guia de los Murcielagos de Argentina. Norman, Oklahoma Museum of Natural History, VIII+119p.

Bianconı, G.V.; R.P. Dı Napoli; D.C. Carneiro \& M. Miretzkı. 2003. A Fazenda Gralha Azul e a conservação dos morcegos da Floresta com Araucária no Paraná. In: IV Encontro Brasileiro para o Estudo de Quirópteros, 2003, Porto Alegre, RS. Divulgações do Museu de Ciências e Tecnologia, Porto Alegre, 2: 62.

BonACCORSO, F.J. 1979. Foraging and reproductive ecology in a panamanian bat community. Bulletin of the Florida State Museum, Biological Sciences, Gainesville, 24 (4): 359-408.

Brosset, A.P. \& P. Charles-Dominique. 1990. The bats from French Guiana: a taxonomic, faunistic and ecological approach. Mammalia, Paris, 54: 509-560.

Brosset, A.; P. Charles-Dominique; A. Cockle; J.F. Cosson \& D. MASSON. 1996. Bat communities and deforestation in French Guiana. Canadian Journal of Zoology, Ottawa, 74: 19741982.

ColweLL, R.K. 2001. Estimates: statistical estimation of species richness and shared speciesfrom samples. Version 6.0.b1. User's Guide and application published at: http:// viceroy.eeb.uconn.edu/estimates.

Cosson, J.; J. Pons \& D. MAsson. 1999. Effects of forest fragmentation on frugivorous and nectarivorus bats in French Guiana. Journal of Tropical Ecology, Cambridge, 15: 515534.

ERICKSON, J.L. \& S.D. WeSt. 2003. Association of bats with local structure and landscape features of forested stands in western Oregon and Washington. Biological Conservation, Essex, 109: 95-102.

Estrada, A. \& R. Coates-Estrada. 2002. Bats in continuos forest, forest fragments and in a agricultural mosaic habitat-island at LosTuxtlas, Mexico. Biological Conservation, Essex, 103: 237-245.

Estrada, A.; R. Coates-estrada \& D. Meritt JR. 1993. Bats species richness and abundance in tropical rain forest fragments and in agricultural habitats at Los Tuxtlas, Mexico. Ecography, Copenhagen, 16: 309-318. 
FÁbian, M.E; A.M. RuI \& K.P. Oliveira. 1999. Distribuição geográfica de morcegos Phyllostomidae (Mammalia, Chiroptera) no Rio Grande do Sul, Brasil. Iheringia, Série Zoologia, Porto Alegre, (87): 143-156.

Félix, J.S.; N.R. dos Reis, I.P. Lima; E.F. Costa \& A.L. Peracchi. 2001. Is the area of the Arthur Thomas Park, with its 82.72 ha, sufficient to maintain viable chiropteran populations? Chiroptera Neotropical, Brasília, 7 (1-2): 129-133.

Fenton, M.B.; L. Acharya; D. Audet; M.B.C. Hickey; C. Merriman; M.K. OBRIst \& D.M. SYME. 1992. Phyllostomid bats (Chiroptera: Phyllostomidae) as indicators of habitat disruption in the Neotropics. Biotropica, Washington, 24 (3): 440-446.

FLEMING, T.H. 1985. Coexistence of five sympatric Piper (Piperaceae) species in a tropical dry forest. Ecology, Durhan, 66 (3): 688-700.

1988. The short-tailed fruit bat: a study in plantanimal interactions. Chicago, University of Chicago Press, $X I I I+365 p$.

Fleming, T.H. \& E.R. Heithaus. 1986. Seasonal foranging behavior of the frugivorous bat Carollia perspicillata. Journal of Mammalogy, Lawrence, 67 (4): 660-671.

GARDNER, A.L. 1977. Feeding habits, p. 293-350. In: J.R. BAKER; J.K. JONES JR. \& D.C. CARTER (Eds). Biology of bats of the New World family Phyllostomidae, part II. Special Publications Museum Texas Tech University, Lubbock, 13: 349.

GonZALEZ, J.C. 1989. Guia para la identificación de los murciélagos del Uruguay. Museu Damaso Antonio Larraña, Serie de Divulgación, Montevideo, 2: 1-50.

Goodwin, G.G. \& A.M. Greenhall. 1961. A review of the bats of Trinidad and Tobago: descriptions, rabies infection and ecology. Bulletin of the American Museum of Natural History, New York, 122 (3): 187-302.

Greenhall, A.M. \& J.L. Paradiso. 1968. Bats and bat banding. Resource Publication, Bureau of Sport Fisheries and Wildlife, United States, 72: 1-48.

HANDLEY JR., C.O \& D.W. MoRRISON. 1991. Foraging behavior, p. 137-140. In: C.O. HANDLEY JR.; D.E. Wilson \& A.L. Gardner. (Eds). Demography and natural history of the common fruit bat, Artibeus jamaicensis, on Barro Colorado Island, Panamá. Smithsonian Contributionsto Zoology, Washington, 511: 1-173.

Handley JR., C.O.; L.L. Gardner \& D.E. Wilson. 1991. Movements, p. 89-130. In: C.O. Handley JR.; D.E. Wilson \& A.L. Gardner (Eds.). Demography and natural history of the common fruit bat, Artibeus jamaicensis, on Barro Colorado Island, Panamá. Smithsonian Contributions to Zoology, Washington, 511: 1-173.

HatSChBACH, G. \& S. Ziller. 1995. Lista vermel ha das plantas ameaçadas de extinção no Estado do Paraná. Curitiba, Instituto Ambiental do Paraná, Deustsche Gesselschaft für Technische Zussammenarbeit, V+139p.

Heithaus, E.R.; T.H. Fleming \& P.A. Opler. 1975. Foraging patterns and resource utilization in seven species of bats in a seasonal tropical forest. Ecology, Durhan, 56: 841-854.

HumphreY, S.R. \& F.J. BonacCCORSO. 1979. Population and community ecology, p. 409-441. In: R.J. BAKER; J.K. JONES JR. \& D.C. Carter (Eds). Biology of bats of the New World family Phyllostomidae, part III. Special Publications Museum Texas Tech University, Lubbock, 16: 1-441.

IBDF (Instituto Brasileiro de Desenvolvimento Florestal). 1981. Plano de Manejo do Parque Nacional do Iguaçu. Brasília, Fundação Brasileira para a Conservação da Natureza, 104p. ITCF. 1987. Plano de manejo do Parque Estadual de Vila Rica do Espírito Santo, Fênix, PR. Curitiba, Instituto de Terras Cartografia e Florestas, IX +86p.

KaLKO, E.K.V. 1997. Diversity in tropical bats, p. 13-43. In: H. ULRICH (Ed.). Tropical biodiversity and systematics. Proceedings of the International Symposium on Biodiversity and Systematics in Tropical Ecosystems, Bonn, 1994. Bonn, Zoologisches Forschungsinstitut und Museum Alexander Koenig.

KoopmAn, K.F. 1993. Order Chiroptera, p. 137-241. In: D.E. WILSON \& D. ReEDER (Eds). Mammals species of the World: a taxonomic and geographic reference. Washington, Smithsoniam Institution Press, $2^{\text {nd }}$ ed., XVIII+1312p.

Kunz, T.H. \& A. KURTA. 1990. Capture methods and holding devices, p. 1-29. In:T.H. Kunz (Ed.). Ecological and behavior methods for the study of bats. Washington, Smithsonian Institution Press, XXII+533p.

Kunz, T.H. \& E.D. Pierson. 1994. Bats of the World: an introduction, p. 1-46. In: R.W. NowAK (Ed.). Walker's bats of the World. Baltimore, The Johns Hopkins University Press, 287p.

KUnZ, T.H. \& P.A. RACEY. 1998. Bat biology and conservation. Washington, Smithsonian Institution Press, XVI+362p.

LAVAL, R.K. 1973. A revision of the neotropical bats of the genus Myotis. Science Bulletin Natural History Museum LoS Angel es County, Los Angeles, 15: 1-53.

LAVAL, R.K. \& H.S. FITCH. 1977. Structure, movements and reproduction in three Costa Rican bat communities. Occasional Papers M useum of Natural History, University of Kansas, Lawrence, 69: 1-27.

LAW, B.S. \& C.R. DICKMAN. 1998. The use of habitat mosaics by terrestrial vertebrate fauna: implications for conservation and management. Biodiversity and Conservation, London, 7: 323-333.

Lim, B.K. \& M.D. Engstrom. 2001. Species diversity of bats (Mammalia: Chiroptera) in Iwokrama Forest, Guyana, and the Guianan subregion: implications for conservation. Biodiversity and Conservation, London, 10: 613-657.

LUDWING, J.A. \& J.F. REYNOLDS. 1988. Statistical ecology: a primer on methods and computing. New York, Wiley, 325p.

MAACK, R. 1981. Geografia física do Estado do Paraná. Rio de Janeiro, J. Olympio, XLIII+442p.

Marinho-Filho, J.S. \& I. Sazima. 1998. Brazilian bats and conservation biology: a first survey, p. 282-294. In: T.H. Kunz

Revista Brasileira de Zoologia 21(4): 943-954, dezembro 2004 
\& P.A. RACEY (Eds). Bat biology and conservation. Washington, Smithsonian Institution Press, XIV +365p.

MIKICH, S.B. 2002. A dieta dos morcegos frugívoros (Mammalia, Chiroptera, Phyllostomidae) de um pequeno remanescente de Floresta Estacional Semidecidual do sul do Brasil. Revista Brasileira de Zoologia, Curitiba, 19 (1): 239-249.

MIKıCH, S.B. \& S.M. SILVA. 2001. Composição florística efenologia das espécies zoocóricas de remanescentes de Floresta Estacional Semidecidual no centro-oeste do Paraná, Brasil. Acta Botânica Brasílica, São Paulo, 15 (1): 89-113.

MIRETZKı, M. 2003. Morcegos do Estado do Paraná, Brasil (Mammalia, Chiroptera): riqueza de espécies, distribuição esíntese do conhecimento atual. Papéis Avul sos de Zoologia, São Paulo, 43 (6): 101-138.

MiretZKı, M. \& T.C.C. Margarido. 1999. Morcegos da Estação Ecológica do Caiuá, Paraná (sul do Brasil). Chiroptera Neotropical, Brasília, 5 (1-2): 105-108.

MulLeR, M.F. \& N.R. Dos ReIS. 1992. Partição de recursos alimentares entre quatro espécies de morcegos frugívoros (Chiroptera, Phyllostomidae). Revista Brasileira de Zoologia, Curitiba, 9 (3/4): 345-355.

Nogueira, M.R. \& A.L. Peracchi. 2002. Fig-seed predation by 2 species of Chiroderma: discovery of a new feeding strategy in bats. Journal of Mammalogy, Lawrence, 84 (1): 225233.

Odum, E.P. 1988. Ecologia. Rio de Janeiro, Editora Guanabara Koogan, XI+434p.

Palmeirim, J.M.; D.L. Gorchov \& S. Stoleson. 1989. Trophic structure of a neotropical frugivore community: is there competition between birds and bats? Oecologia, Berlin, 79: 403411.

Passos, F.C.; Silva, W.R.; Pedro, W.A. \& M.R. Bonin. 2003. Frugivoria em morcegos (Mammalia, Chiroptera) no Parque Estadual de Intervales, sudeste do Brasil. Revista Brasileira de Zoologia, Curitiba, 20 (3): 511-517.

Patterson, B. \& R. Pascual. 1972. The fossil mammal fauna of South America, p. 247-309. In: A. KeAst; F.C. ERK \& B. Glass (Eds). Evolution, mammals and southern continents. Albany, State University New York Press, 543p.

Pedro, W.A.; M.P. Geraldes; G.G. Lopez \& C.J.R. Alho. 1995. Fragmentação de hábitat e a estrutura de uma taxocenose de morcegos em São Paulo (Brasil). Chiroptera Neotropical, Brasília, 1 (1): 4-6.

Pedro, W.A. \& V.A. TAddel. 1997. Taxonomic assemblage of bats from Panga Reserve, southeastern Brazil: abundance patterns and trophic relations in the Phyllostomidae (Chiroptera). Boletim do M useu de Biologia Prof. Mello Leitão, n. ser., Santa Teresa, 6: 3-21.

Pedro, W.A; C. de Carvalho; M.M. Hayashi; A. Bredt; N.M.S. Armani; M.M.S. Silva; L. Gomes; C.A. Gonçalves \& N.F. Peres. 1997. Notes on Vampyressa pusilla (Wagner, 1843) in the south of São Paulo State. Chiroptera Neotropical, Brasília, 3 (2): 79-80.
Pedro, W.A.; F.C. PAssos \& B.K. Lim. 2001. Morcegos (Chiroptera; Mammalia) da Estação Ecológica de Caetetus, Estado de São Paulo. Chiroptera Neotropical, Brasília, 7 (1-2): 136-140.

Quadros, J.; N.C. Cáceres; L.M. Tiepolo \& M.S. Wängler. 2000. Mastofauna do Parque Estadual do Rio Guarani e área de influência da Usina Hidrelétrica de Salto Caxias, baixo rio Iguaçu, Estado do Paraná, p. 822-829. In: M.S. MILANo \& V. Theulen (Eds). Campo Grande, II Congresso Brasileiro de Unidades de Conservação, Anais, XVI +854p.

ReIs, N.R. DOS \& A.L. PeRACCHI. 1987. Quirópteros da região de Manaus, Amazonas, Brasil (Mammalia, Chiroptera). Bolletim do Museu Paraense Emilio Goeldi, Zoologia, Belém, 3 (2): 161-182.

Reis, N.R. dos; A.L. Peracchi \& M.K. Onukı. 1993a. Quirópteros de Londrina, Paraná, Brasil (Mammalia, Chiroptera). Revista Brasileira de Zoologia, Curitiba, 10 (3): 371-381.

Reis, N.R. dos; M.F. Muller; E.S. Soares \& A.L. Peracchi. 1993b. Lista e chave de quirópteros do Parque Estadual Mata dos Godoy e arredores, Londrina, PR. Semina, 14 (2): 120-126.

ReIS, N.R. Dos \& M.F. MuLler. 1995. Bat diversity of forests and open areas in a subtropical region of South Brazil. Ecologia Austral, Córdoba, 5: 31-36.

Reis, N.R. dos; A.L. Peracchi; M.F. Muller; E.A. Bastos \& E.S. SoARES. 1996. Quirópteros do Parque Estadual Morro do Diabo, São Paulo, Brasil (Mammalia, Chiroptera). Revista Brasileira de Biologia, Rio de Janerio, 56 (1): 87-92.

Reis, N.R. dos; A.L. Peracchi \& M.L. Sekiama. 1999. Morcegos da Fazenda M onteAlegre, Telêmaco Borba, Paraná (Mammalia, Chiroptera). Revista Brasileira de Zoologia, Curitiba, 16 (2): 501-505.

Reis, N.R. dos; A.L. Peracchi; M.L. Sekiama \& I.P. Lima. 2000. Diversidade de morcegos (Chiroptera, Mammalia) em fragmentos florestais no Estado do Paraná, Brasil. Revista Brasileira de Zoologia, Curitiba, 17 (3): 697-704.

ReIs, N.R. dos; A.L. Peracchi \& I.P. LimA. 2002. Morcegos da bacia do rio Tibagi, p. 251-270. In: M.E. MedRI; E. BianchinI; O.A. Shibatta \& J.A. Pimenta (Eds). A bacia do rio Tibagi. Londrina, Edição dos Editores, 595p.

Reis, N.R. dos; M.L.S. Barbieri; I.P. Lima \& A.L. Peracchi. 2003. O que é melhor para manter a riqueza de espécies de morcegos (Mammalia, Chiroptera): um fragmento florestal grande ou vários fragmentos de pequeno tamanho? Revista Brasileira de Zoologia, Curitiba, 20 (2): 225-230.

RidLEY, H.N. 1930. The dispersal of plants throughout the world. Ashford, England, L. Reeve, 744p.

RUI, A.M. \& M.E. FÁBIAN. 1997. Quiropteros dela familia Phyllostomidae (Mammalia, Chiroptera) en selvas del estado de Rio Grande do Sul, Brasil. Chiroptera Neotropical, Brasília, 3 (2): 75-77.

RuI, A.M.; M.E. FÁbIAn \& J.O. Meneghetı. 1999. Distribuição geográfica e análise morfológica de Artibeus lituratus Olfers e de Artibeus fimbriatus Gray (Chiroptera, Phyllostomidae) no Rio Grande do Sul, Brasil. Revista Brasileira de Zoologia, 
Curitiba, 16 (2): 447-460.

Schulze, M.D.; N.E. Seavy \& D.F. Whitacre. 2000. A comparison of the phyllostomid bat assemblages in undisturbed neotropical forest and in forest fragments of a Slash-and-Burn farming mosaic in Petén, Guatemala. Biotropica, Washington, 32 (1): 174-184.

Sekiama, M.L.; N.R. Reis; A.L. Perachi \& V.J. Rocha. 2001. Morcegos do Parque Nacional do Iguaçu, Paraná (Chiroptera, Mammalia). Revista Brasileira de Zoologia, Curitiba, 18 (3): 749-754.

Silveira-Neto, S.O.; D. Nakano \& N.A.V. Nova. 1976. Manual de ecologia dos insetos. São Paulo, Ed. Agronômica Ceres, 419p.

Simmons, N.B. \& R.S. Voss. 1998. The mammals of Paracou, French Guiana: a neotropical lowdland rainforest fauna. part 1. Bats. Bulletin of the American Museum of Natural History, New York, 237: 1-219.

SIPISNKI, E.A.B. \& N.R. ReIs. 1995. Dados ecológicos dos quirópteros da Reserva Volta Velha, Itapoá, Santa Catarina, Brasil. Revista Brasileira de Zoologia, Curitiba, 12 (3): 519-528.

SToCKWELL, E.F. 2001. Morphology and flight manoeuvrability in New World leaf-nosed bats (Chiroptera: Phyllostomidae). Journal of Zoology, London, 254: 505-514.

Straube, F.C. \& G.V. BiAnConı. 2002. Sobre a grandeza e a unidade utilizada para estimar esforço de captura com utilização deredes-deneblina. Chiroptera Neotropical, Brasília, 8 (12): $150-152$.

Taddel, V.A. \& W.A. Pedro. 1998. Morcegos (Chiroptera, Mammalia) do Vale do Ribeira, Estado de São Paulo: diversidade de espécies. São Carlos. In: Anais VIII Seminário Regional de Ecologia, p. 911-919.

Taddel, V.A.; C.A. Nobile \& E. M orielle-Versute. 1998. Distribuição geográfica e análise morfométrica comparativa em Artibeus obscurus (Schinz, 1821) e Artibeus fimbriatus Gray, 1838 (Mammalia, Chiroptera, Phyllostomidae). Ensaios e Ciências, Campo Grande, 2 (2): 49-70.

Recebido em 13.IV.2004; aceito em 17.XI.2004.
Tıмm, R.M. 1994. The mammal fauna, p. 229-237. In: L.A. McDade; K.S. BAWA; H.A. Hespenheide \& G.S. HaRTSHORn (Eds). La Selva: Ecology and natural history of a neotropical rain forest. Chicago, University of Chicago Press, 486p.

Trajano, E. 1984. Ecologia de populações de morcegos cavernícolas em uma região cárstica do sudeste do Brasil. Revista Brasileira de Zoologia, Curitiba, 2 (5): 255-320.

TRIOLA, M.F. 1999. Introdução à Estatística. Rio de Janeiro, Livros Técnicos e Científicos Editora, 7ạ ed., 410p.

VAN DER PIJL, L. 1957. The dispersal of plants by bats (Chiropterochory). Acta Botanica Neerlandica, Amsterdam, 6: 291-315.

Veloso, H.P.; L.C. Oliveira-Filho; A.M.S.F. VaZ; M.P.M. Lima; R. Marquete \& J.E.M. BrazÃo. 1992. Manual técnico da vegetação brasileira. Manuais técnicos em geociências, 1 . Rio deJaneiro, Secretaria de Planejamento, Orçamento e Coordenação, Fundação Instituto Brasileiro de Geografia e Estatística, Diretoria de Geociências, Departamento de Recursos Naturais e Estudos Ambientais, 93p.

VizotTo, L.D. \& V.A. TADDEı. 1973. Chave para determinação de quirópteros brasileiros. São José do Rio Preto, Gráfica Francal, 72p.

VonHOF, M.J. \& R.M.R. BARCLAY. 1996. Roost site selection and roosting ecology of forest-dwelling bats in southern British Columbia. Canadian Journal of Zoology, Otawa, 74: 17971805.

Wendeln, M.C.; J.R. Rankle \& E.K.V. Kalko. 2000. Nutritional values of 14 species of figs and bat feeding preferences in Panama. Biotropica, Washington, 32: 489- 501.

WILSON, D.E.; C.F. AscorRA \& S. SolARI. 1996. Bats as indicators of habitat disturbance, p. 613-625. In: D.E. WILSON \& A. SAndoval (Eds). Manu: The biodiversity of southeastern Peru. Washington, Office of biodiversity programs. National Museum of Natural History, Smithsonian Institution, 365p.

ZortÉA, M. \& A.G. Chiarello. 1994. Observations on the big fruit-eating bat, Artibeus lituratus, in urban reserve of southeast Brazil. Mammalia, Paris, 58 (4): 665-670.

Revista Brasileira de Zoologia 21(4): 943-954, dezembro 2004 